\title{
Preliminary Computational Framework to Map MRI-Derived Markers to predict Response to Cardiac Resynchronization Therapy
}

\author{
Carolina Vallecilla ${ }^{1}$, Martino Alessandrini ${ }^{1}$, Claudio Fabbri ${ }^{1}$, Corrado Tomasi ${ }^{2}$, Cristiana Corsi $^{1}$, \\ Stefano Severi ${ }^{1}$ \\ ${ }^{1}$ University of Bologna, Cesena, Italy \\ ${ }^{2}$ Santa Maria delle Croci Hospital, Ravenna, Italy
}

\begin{abstract}
Prediction of the response to cardiac resynchronization therapy (CRT) is still uncertain. On our previous CRT clinical research, we have found that a decrease in the ratio between the two principal axes of the $3 D$ trajectory of the electrode at the pacing site $\left(S_{1} / S_{2}\right)$ recorded before and after pacing could define a marker between responders and non-responders to CRT. The aim of this work is to design a framework to map the $S_{1} / S_{2}$ marker on the $3 D$ ventricular anatomy as a preliminary test to verify if the concept of the $S_{1} / S_{2}$ may predict the response to CRT in a pre-implant scenario. Based on MR images of a CRT candidate, the $3 D$ mesh of the left ventricle geometry is constructed. Using image registration we are able to track the deformation of the mesh throughout the cardiac cycle and to compute the trajectory of each point of the mesh. Then the $S_{1} / S_{2}$ is calculated for every trajectory and mapped on a $3 D$ geometry representation. We have applied this framework to one CRT patient, highlighting that in the area in which the electrode was placed the $S_{1} / S_{2}$ was low. This value suggests a poor possibility of a pacing-induced decrease for the $S_{1} / S_{2}$ ratio after implant. Consistently the patient was classified as non-responder at the clinical follow-up. Ongoing work focuses on the clinical validation of $S_{1} / S_{2}$ as a tool for the prediction of CRT response and the acquisition of MR data of potential candidates to CRT for the assessment of the presented framework.
\end{abstract}

\section{Introduction}

Cardiac resynchronization therapy (CRT) is an established treatment in heart failure (HF) and it has been proved highly effective in improving left ventricular (LV) function and survival in selected cases with HF. Unfortunately the relations between left ventricular (LV) dynamics and LV intravenous pace are still a matter of investigation since about 30 to $50 \%$ of patients do not respond favourably to this therapy [1-3]. CRT outcome allegedly depends on multiple factors, such as myocardial structure, arrhythmias, dyssynchrony, intravenous lead placement, device functioning, comorbidities, and other medical treatments [4]. These factors and their interactions have been widely investigated to understand which might contribute to improve CRT success rate. In our previous work, we studied the coronary sinus (CS) lead tip movements as a source of information about acute resynchronization of LV mechanics in CRT recipients $[5,6]$. We evaluated the acute effects of biventricular pacing $(\mathrm{BiV})$ at implant through the reconstruction of the three-dimensional (3D) geometric CS lead tip trajectory throughout the cardiac cycle via a fluoroscopy-based method specifically designed and developed [5]. In a preliminary testing on 22 patients we showed that modifications of the $3 \mathrm{D}$ reconstruction of CS lead tip trajectory induced acutely by $\mathrm{BiV}$ were related to resynchronization and predicted long term volumetric response to CRT, defined as a $>15 \%$ reduction in the echocardiographic end systolic volume at 6-month followup in comparison with pre-implant status [6]. Research to confirm these results on a multi-centric population of one hundred CRT patients is ongoing.

The index able to discriminate between responder (R) and non-responder (NR) patients was the change in the ratio between the two principal axes of this 3D trajectory $\left(\mathrm{S}_{1} / \mathrm{S}_{2}\right)$ recorded as a delta before and after pacing start. We found that NR subjects would be characterized by low values of $S_{1} / S_{2}$ (i.e. more elongated trajectory) that after pacing do not increase towards higher values. A possible interpretation is that in these cases the myocardial wall motion responds to a local pattern of contraction and not to a global (more synchronized) one, as desired.

In $[5,6]$ the ratio $S_{1} / S_{2}$ was computed for the trajectory of a specific anatomical point in which the CS lead tip was located during the device implant; the analysis was performed using two fluoroscopic planar views and the 3D trajectory was reconstructed applying stereo photogrammetric rules. The aim of this work is to design a framework to map the $S_{1} / S_{2}$ marker, on the whole 3D ventricular anatomy as a preliminary test to verify if the 


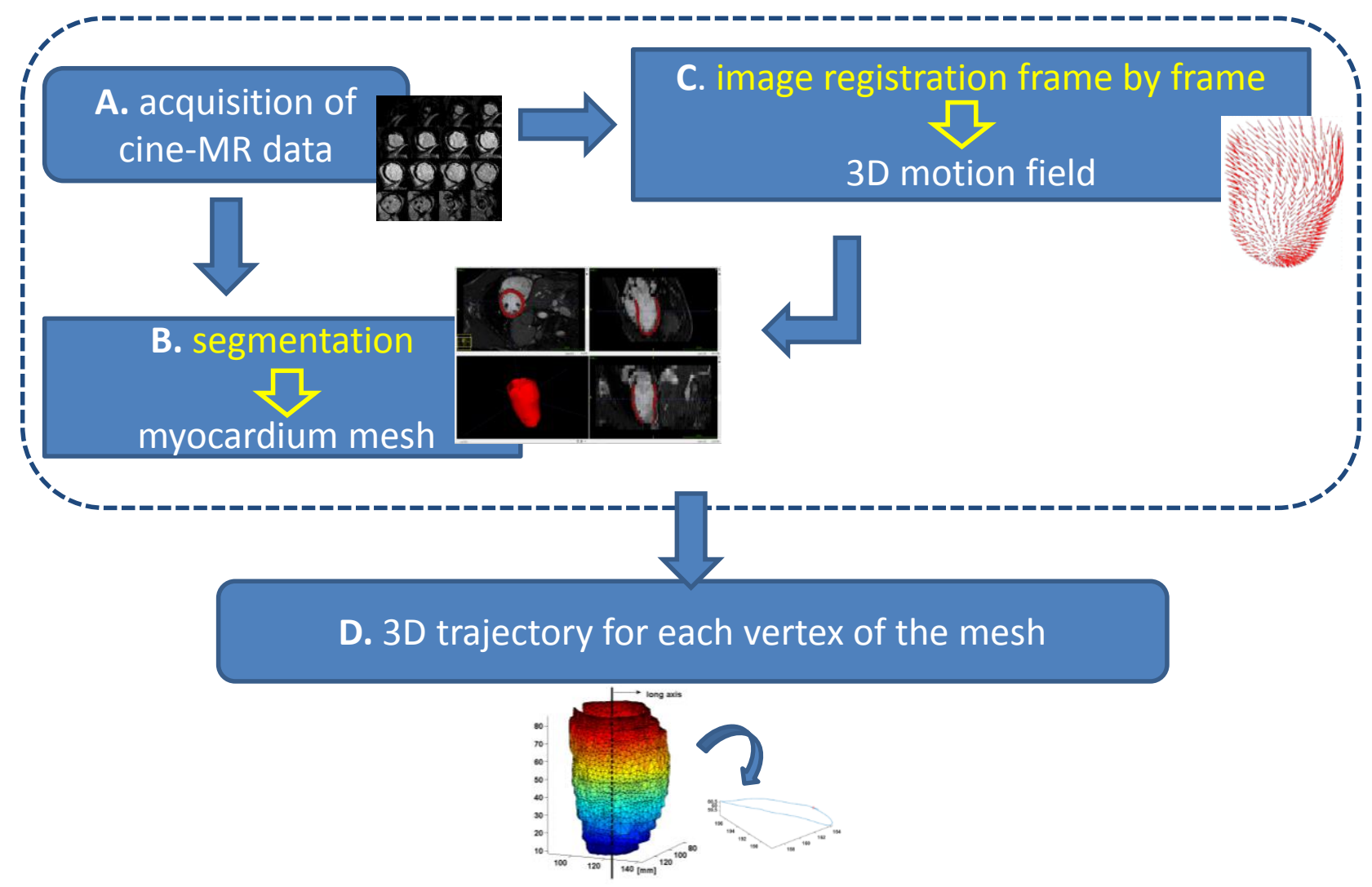

Figure 1. Schematic workflow of the 3D trajectory computation algorithm.

concept of the $S_{1} / S_{2}$ may predict the response to CRT in a realistic 3D pre implant scenario.

\section{Methods}

The workflow of the developed procedure to derive the 3D trajectory of each vertex of the left ventricular (LV) endocardial and epicardial surfaces throughout the cardiac cycle, is shown in Figure 1.

In one patient, images were obtained by using a $1.5 \mathrm{~T}$ MRI scanner (Philips Medical System, Achieva). ECGtriggered images were acquired in breath hold (echo time: $1.53 \mathrm{~ms}$, repetition time: $3.05 \mathrm{~ms}$, flip angle: $60^{\circ}$, in-plane resolution $0.94 \times 0.94 \mathrm{~mm}$ and slice thickness $7 \mathrm{~mm}$ with no gap between slices, image size of $432 \times 432$ pixels) at a temporal resolution of 25 frames per cardiac cycle (Figure 1.A).

Segmentation of endocardial and epicardial boundaries of the LV were automatically obtained applying a previously developed and validated algorithm based on probabilistic level set model [7]. The algorithm was applied to the first acquired MRI volume, corresponding to the end diastolic (ED) phase. This step resulted in a binary 3D mask that was converted in in a mesh (Figure 1.B).

In order to extract the patient specific LV motion over the cardiac cycle, we registered the MR volumes. Hereto, the displacement $\mathbf{d}_{\mathrm{i}_{\rightarrow+1}+1}(\mathbf{x})$ between two successive MRI volumes $I_{i}(\mathbf{x})$ and $I_{i+1}(\mathbf{x})$ was computed by elastic image registration. In order to initialize the non-rigid registration problem, we chose to perform a rigid transformation first. The result $\mathbf{T}_{0}$ is combined with a non rigid transformation $\mathbf{T}^{\mathrm{NR}}$ as specified in the following:

$$
\mathrm{T}(\mathbf{x})=\mathrm{T}^{\mathrm{NR}}\left(\mathrm{T}_{0}(\mathbf{x})\right)=\left(\mathrm{T}^{\mathrm{NR}} \circ \mathrm{T}_{0}\right)(\mathbf{x})
$$

As a similarity measure for the mono-modality registration we used the mean square difference. The global displacement of a general volume at time $\mathrm{i}$ with respect to the reference volume at time 0 was then computed by accumulating the successive inter-frame displacement estimates by means of the recursive formula $\mathbf{d}_{\mathrm{i} \rightarrow 0}(\mathbf{x})=\mathbf{d}_{\mathrm{i} \rightarrow \mathrm{i}-1}(\mathbf{x}) \circ \mathbf{d}_{\mathrm{i}-1 \rightarrow 0}(\mathbf{x})$ with $\mathbf{d}_{0 \rightarrow 0}(\mathbf{x})=0$.

The deformation displacement field (Figure 1.C) was then used to propagate the LV mesh over the full cardiac cycle. By calling $\mathbf{x}_{0}$ the position of a mesh vertex at time 0 , we computed its position $\mathbf{x}_{\mathrm{i}}$ at time $\mathrm{i}$ by sampling the computed displacement field, $\mathbf{x}_{\mathrm{i}}=\mathbf{x}_{0}+\mathbf{d}_{\mathrm{i} \rightarrow 0}\left(\mathbf{x}_{0}\right)$. As such, a set of $\mathrm{N}$ tetrahedral meshes representing the instantaneous position of the LV on each available MRI volume was obtained. 
The computation of the 3D trajectory throughout the cardiac cycle for each vertex of the mesh was straightforward (Figure 1.D). Then the ratio $S_{1} / S_{2}$ was calculated for each vertex trajectory throughout the cardiac cycle and mapped on a 3D geometry representation.

In addition, for strain analysis, all the surfaces were realigned with respect to the long axis position in each volume throughout the cardiac cycle. For each vertex $v$, strain $\mathrm{S}$ was computed as:

$$
S_{t}(v)=\frac{\mathrm{l}-L_{0}}{L_{0}}
$$

where $L_{0}$ is the distance between the long axis and the vertex $v$ at ED and 1 is the distance between the long axis and the same vertex $v$ in the current time frame $t$. Mean strain value was then computed for each region of the LV [8].

This framework was applied to MRI data acquired in one normal subject and in one patient before the CRT device implant.

\section{Results}

Three orthogonal planes of the MRI data at ED with the myocardium segmentation superimposed is shown in Figure 2.

The 3D map of the parameter $S_{1} / S_{2}$ in the CRT patient is shown in Figure 3.

In the CRT patient, the ratio $S_{1} / S_{2}$ in the pacing site region was quite low, 1.52, representative of a trajectory spanning both the two principal directions (Figure 4). With respect to the neighbouring territories the pacing electrode was located in a region whose movement is quite homogeneous. At six month follow-up this patient was classified as non-responder.

Strain analysis clearly showed important differences between the normal subject and the CRT patient in both strain amplitude and strain curve trend (Figure 5).

\section{Conclusion}

These preliminary results suggest the parameter $S_{1} / S_{2}$ may represent an effective index to evaluate coronary territories more suitable for CRT stimulation, by identifying desynchronized LV wall area. This indication may be related with the theory that correction or abolishment of ventricular mechanical dissynchrony supports the reversal of left ventricular remodeling and improve CRT rate of success.

Ongoing research focuses on the clinical validation of $\mathrm{S}_{1} / \mathrm{S}_{2}$ as a tool for the prediction of the acute CRT response and the acquisition of MR data of potential candidates to CRT for the assessment of the framework.

The introduction of $S_{1} / S_{2}$ as a tool for the prediction of CRT response could help to identify CRT candidates or to plan the procedure, identifying the optimal zones for $\mathrm{v}-\mathrm{V}$
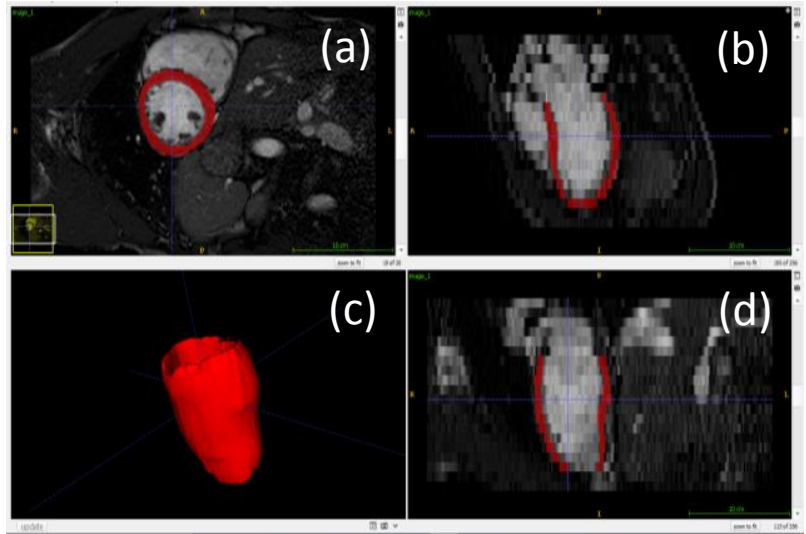

Figure 2. Short-axis (a), 4-chamber (b) and 2-chamber (d) views of the MRI data at ED with the myocardium segmentation (c) superimposed.

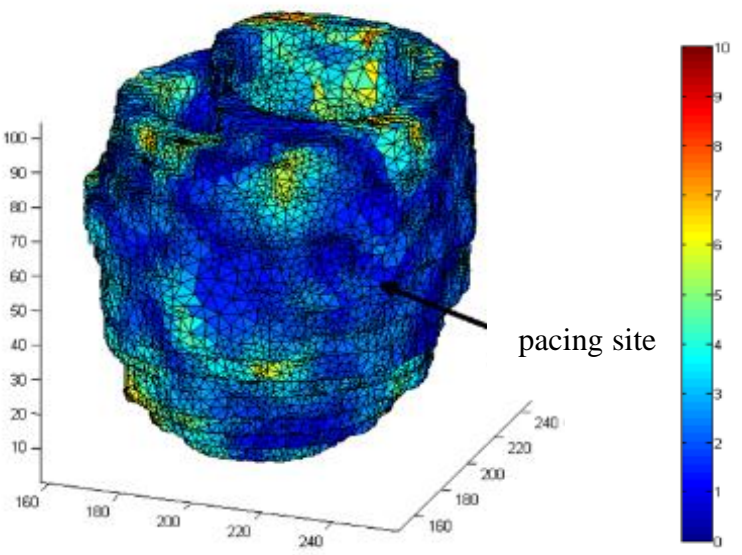

Figure 3. Example of a 3D map of the index $S_{1} / S_{2}$ in the CRT patient, with the indication of the pacing site region.

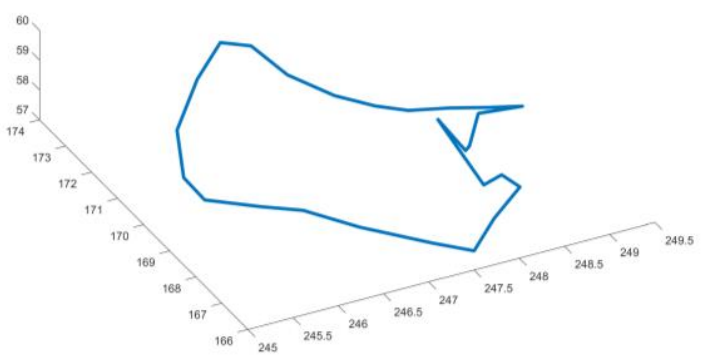

Figure 4. Computed trajectory at the pacing site.

pacing in a non-invasive manner previous to intervention. 


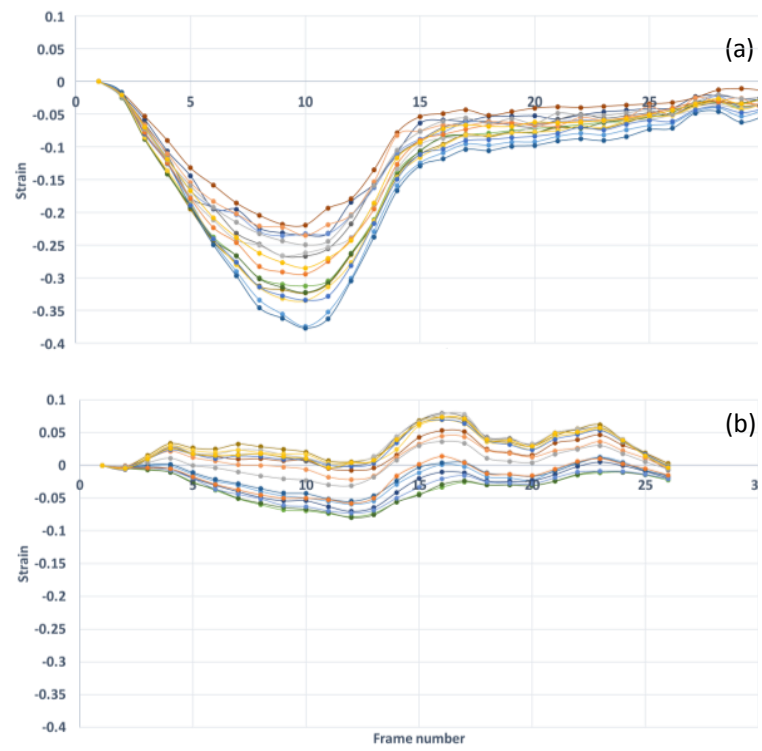

Figure 5. Strain curves in the normal subject (a) and in the CRT patient (b).

\section{References}

[1] Bristow MR, Saxon LA, Boehmer J, et al. for the Comparison of Medical Therapy, Pacing, and Defibrillation in Heart Failure (COMPANION) Investigators. Cardiac resynchronization therapy with or without an implantable defibrillator in advanced chronic heart failure. N Engl J Med, 2004;350:2140-2150.

[2] Cleland JG, Daubert JC, Erdmann E, et al. Cardiac Resynchronization-Heart Failure (CARE-HF) Study Investigators. The effect of cardiac resynchronization on morbidity and mortality in heart failure. N Engl J Med, 2005;352:1539-1549.
[3] Moss AJ, Hall WJ, Cannom DS, et al. MADIT-CRT Trial Investigators. Cardiac-resynchronization therapy for the prevention of heart-failure events. $\mathrm{N}$ Engl $\mathrm{J}$ Med, 2009;361:1329-1338.

[4] Verbrugge FH, Dupont M, Rivero-Ayerza M, et al. Comorbidity significantly affects clinical outcome after cardiac resynchronization therapy regardless of ventricular remodeling. J Card Fail, 2012;18:845-853.

[5] Corsi C, Tomasi C, Turco D, et al. 3D dynamic position assessment of the coronary sinus lead in cardiac resynchronization therapy. Med Biol Eng Comput, 2011;49:901-908.

[6] Tomasi C, Corsi C, Turco D, et al. An exploratory study on coronary sinus lead tip trajectory changes in cardiac resynchronization therapy, Heart Rhythm, 2013;10(9):13601367.

[7] Corsi C, Veronesi F, Lambert C et al. Automated Frame-byFrame Endocardial Border Detection from Cardiac Magnetic Resonance Images for Quantitative Assessment of Left Ventricular Function: Validation and Clinical Feasibility. Journal of Magnetic Resonance Imaging, 2009;29(3):560568.

[8] Cerqueira MD, Weissman NJ, Dilsizian V, et al. Standardized myocardial segmentation and nomenclature for tomographic imaging of the heart: a statement for healthcare professionals from the Cardiac Imaging Committee of the Council on Clinical Cardiology of the American Heart Association. Circulation 2002;105:539-542.

Address for correspondence.

Stefano Severi

DEI, University of Bologna,

Viale Risorgimento 2, 40136, Bologna, Italy stefano.severi@unibo.it 